\title{
DNA Sequence Modulates Conformation of the Food Mutagen 2-Amino-3- methylimidazo[4,5-f]quinoline (IQ) in the Recognition Sequence of the NarI Restriction Enzyme
}

Supporting Information

$\underline{\text { Revised Manuscript }}$

Feng Wang, C. Eric Elmquist, James S. Stover, Carmelo J. Rizzo* and Michael P. Stone*

Department of Chemistry, Center in Molecular Toxicology, Vanderbilt-Ingram Cancer Center, Vanderbilt University, Nashville, Tennessee 37235

* Authors to whom correspondence should be addressed. Department of Chemistry, Center in Molecular Toxicology, and Vanderbilt-Ingram Cancer Center, Vanderbilt University, Nashville, TN 37235

Michael P. Stone, telephone 615-322-2589; FAX 615-322-7591;

email michael.p.stone@vanderbilt.edu

Carmelo J. Rizzo, telephone 615-322-6100, FAX 615-343-1234,

email carmelo.rizzo@vanderbilt.edu

Running Title: C8-dG IQ DNA Adduct

$\dagger$ This work was supported by NIH grant CA-55678 (M.P.S.). The Vanderbilt Center for Molecular Toxicology is funded by a center grant from the National Institute of Environmental Health Sciences (ES-00267). J.S.S. was supported by NIH pre-doctoral traineeship ES-07028. Funding for the NMR spectrometers was supplied by NIH Grants RR-05805, ES-00267, and Vanderbilt University. 
Wang, F., et al.

Table S1. $\quad{ }^{1}$ H Chemical Shift Assignments of the Non-exchangeable Protons of the IQModified NarIIQ1 and NarIIQ2 Duplexes (ppm).

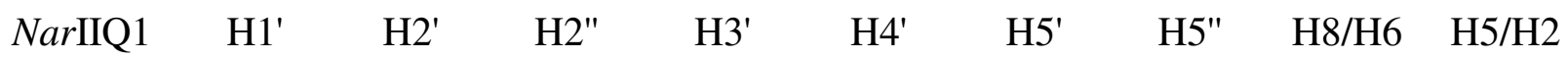

$\begin{array}{llllllllll}\mathrm{C}^{1} & 5.74 & 2.33 & 2.57 & 4.64 & 3.99 & 3.99 & 3.76 & 7.90 & 5.82\end{array}$

$\begin{array}{lllllllll}\mathrm{T}^{2} & 5.99 & 2.03 & 2.56 & 4.90 & 4.17 & 3.99 & 3.99 & 7.69\end{array}$

$\begin{array}{lllllllll}\mathrm{C}^{3} & 5.88 & 1.97 & 2.53 & 4.82 & 4.22 & 4.02 & 7.32 & 5.48\end{array}$

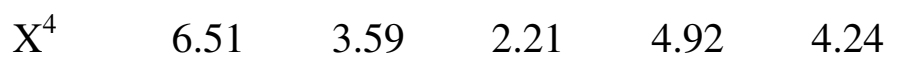

$\begin{array}{lllllllll}\mathrm{G}^{5} & 5.73 & 2.35 & 2.47 & 4.97 & 4.37 & 4.13 & 3.88 & 7.77\end{array}$

$\begin{array}{llllllllll}\mathrm{C}^{6} & 5.59 & 1.87 & 2.27 & 4.85 & 4.06 & 4.87 & 4.02 & 7.23 & 5.07\end{array}$

$\begin{array}{llllll}\mathrm{G}^{7} & 5.69 & 2.49 & 2.55 & 4.83 & 4.31\end{array}$

$\begin{array}{llllllllll}\mathrm{C}^{8} & 5.76 & 1.92 & 2.29 & 4.75 & 4.06 & 3.92 & 3.99 & 7.29 & 5.17\end{array}$

$\begin{array}{llllllllll}\mathrm{C}^{9} & 5.30 & 1.91 & 2.37 & 4.80 & 3.92 & 3.97 & 3.93 & 7.47 & 5.46\end{array}$

$\begin{array}{llllllllll}\mathrm{A}^{10} & 6.16 & 2.69 & 2.91 & 4.99 & 4.30 & 4.05 & 4.18 & 8.32 & 7.72\end{array}$

$\begin{array}{llllllll}\mathrm{T}^{11} & 5.89 & 2.00 & 2.43 & 4.82 & 4.70 & 4.02 & 7.19\end{array}$

$\begin{array}{llllllll}\mathrm{C}^{12} & 6.13 & 2.10 & 2.15 & 4.54 & 3.88 & 7.59 & 5.55\end{array}$

$\begin{array}{lllllllll}\mathrm{G}^{13} & 5.53 & 2.13 & 2.65 & 4.84 & 4.17 & 3.57 & 3.68 & 7.89\end{array}$

$\begin{array}{llllllllll}\mathrm{A}^{14} & 6.18 & 2.72 & 2.93 & 4.92 & 4.35 & 4.01 & 4.14 & 8.31 & 7.93\end{array}$

$\begin{array}{llllllll}\mathrm{T}^{15} & 5.57 & 1.82 & 2.21 & 4.92 & 4.02 & 3.91 & 7.08\end{array}$

$\begin{array}{llllllll}\mathrm{G}^{16} & 5.50 & 2.48 & 2.58 & 4.94 & 4.22 & 3.98 & 7.76\end{array}$

$\begin{array}{llllll}\mathrm{G}^{17} & 5.69 & 2.39 & 2.52 & 4.84 & 4.36\end{array}$

$\begin{array}{llllllll}\mathrm{C}^{18} & 5.47 & 1.81 & 2.21 & 4.82 & 3.99 & 7.20 & 5.08\end{array}$

$\begin{array}{lllllllll}\mathrm{G}^{19} & 5.73 & 2.46 & 2.57 & 5.06 & 4.26 & 3.99 & 3.98 & 7.77\end{array}$

$\begin{array}{lllllllll}\mathrm{C}^{20} & 5.82 & 1.82 & 2.20 & 4.87 & 4.14 & 4.08 & 6.85 & 4.97\end{array}$

$\begin{array}{llllllllll}\mathrm{C}^{21} & 6.42 & 2.30 & 2.60 & 4.95 & 4.54 & 4.05 & 4.14 & 7.99 & 6.09\end{array}$

$\begin{array}{lllllllll}\mathrm{G}^{22} & 5.06 & 2.04 & 2.34 & 4.80 & 4.33 & 3.94 & 4.23 & 7.40\end{array}$ 
Wang, F., et al.

$\begin{array}{llllllllll}\mathrm{A}^{23} & 5.89 & 2.44 & 2.71 & 4.94 & 4.34 & 4.23 & & 7.94 & 7.66 \\ \mathrm{G}^{24} & 5.85 & 2.23 & 2.11 & 4.57 & 4.33 & 3.87 & 4.00 & 7.54\end{array}$


Wang, F., et al.

\begin{tabular}{|c|c|c|c|c|c|c|c|c|c|}
\hline NarIIQ2 & $\mathrm{H} 1^{\prime}$ & $\mathrm{H} 2^{\prime}$ & H2" & H3' & $\mathrm{H} 4{ }^{\prime}$ & H5' & H5" & H8/H6 & $\mathrm{H} 5 / \mathrm{H} 2$ \\
\hline $\mathrm{C}^{1}$ & 5.80 & 1.95 & 2.30 & 4.47 & 4.54 & 3.71 & 3.66 & 7.86 & 5.80 \\
\hline $\mathrm{T}^{2}$ & 6.08 & 2.00 & 2.31 & 4.54 & 4.12 & 3.85 & & 7.55 & 6.08 \\
\hline$C^{3}$ & 5.37 & 1.93 & 2.13 & 4.73 & 3.95 & 3.87 & 3.87 & 7.47 & 5.37 \\
\hline $\mathrm{G}^{4}$ & 5.94 & 2.00 & 2.85 & 4.91 & 4.14 & & 3.83 & 7.45 & 5.94 \\
\hline$X^{5}$ & 5.97 & 3.57 & 2.06 & 4.73 & 4.07 & & & 7.73 & 5.97 \\
\hline$C^{6}$ & 5.73 & 1.72 & 2.21 & 4.73 & 4.04 & & 3.40 & 7.34 & 5.73 \\
\hline $\mathrm{G}^{7}$ & 5.68 & 2.21 & 2.44 & 4.85 & & 4.21 & & 7.81 & 5.68 \\
\hline$C^{8}$ & 5.82 & 1.82 & 2.21 & 4.67 & 4.04 & 4.05 & & 7.25 & 5.82 \\
\hline$C^{9}$ & 5.29 & 1.92 & 2.19 & 4.70 & 4.70 & 3.87 & 7.42 & 5.51 & 5.29 \\
\hline$A^{10}$ & 6.23 & 2.45 & 2.73 & 4.84 & 4.23 & 4.02 & 3.95 & 8.32 & 6.23 \\
\hline $\mathrm{T}^{11}$ & 5.93 & 1.73 & 2.16 & 4.67 & 4.19 & 4.02 & & 7.13 & 5.93 \\
\hline$C^{12}$ & 6.20 & 2.02 & 2.06 & 4.40 & 4.45 & 3.87 & 3.87 & 7.51 & 6.20 \\
\hline $\mathrm{G}^{13}$ & 5.60 & 2.41 & 2.59 & 4.68 & 4.07 & 3.55 & & 7.89 & 5.60 \\
\hline $\mathrm{A}^{14}$ & 6.24 & 2.49 & 2.70 & 4.88 & 4.29 & 4.09 & 4.01 & 8.30 & 6.24 \\
\hline $\mathrm{T}^{15}$ & 5.62 & 1.71 & 2.12 & 4.74 & 4.45 & 4.15 & & 7.02 & 5.62 \\
\hline $\mathrm{G}^{16}$ & 5.51 & 2.42 & 2.50 & 4.88 & 4.14 & 4.22 & & 7.73 & 5.51 \\
\hline $\mathrm{G}^{17}$ & 5.74 & 2.31 & 2.47 & 4.87 & 4.26 & & & 7.63 & 5.74 \\
\hline$C^{18}$ & 5.45 & 1.66 & 2.03 & 4.64 & 3.96 & & & 7.10 & 5.45 \\
\hline $\mathrm{G}^{19}$ & 5.69 & 2.45 & 2.54 & 4.92 & 4.21 & 3.98 & 3.85 & 7.56 & 5.69 \\
\hline$C^{20}$ & 6.38 & 2.22 & 2.49 & 4.95 & 4.41 & 4.21 & 4.03 & 7.98 & 6.38 \\
\hline$C^{21}$ & 5.31 & 1.15 & 1.88 & 4.53 & 4.05 & 4.12 & 3.96 & 7.09 & 5.31 \\
\hline $\mathrm{G}^{22}$ & 5.08 & 2.44 & 2.49 & 4.60 & 4.12 & 4.12 & & 7.82 & 5.08 \\
\hline$A^{23}$ & 6.05 & 2.47 & 2.65 & 4.85 & 4.30 & & & 8.05 & 6.05 \\
\hline $\mathrm{G}^{24}$ & 5.91 & 2.17 & 2.02 & 4.80 & 4.49 & 3.99 & & 7.57 & 5.91 \\
\hline
\end{tabular}


Table S2. Chemical Shift assignments (ppm) of the Exchangeable Imino and Amino Protons of the NarIIQ1 and NarIIQ2 Duplexes.

$\begin{array}{llll}\text { NarIIQ1 } & \text { base pair } & \mathrm{G} \text { N1H/T N3H C N4Ha amino } & \mathrm{C} \text { N4Hb amino } \\ \mathrm{T}^{2} \bullet \mathrm{A}^{23} & 13.97 & & \\ \mathrm{C}^{3} \bullet \mathrm{G}^{22} & 12.62 & 7.24 & 8.83 \\ \mathrm{X}^{4} \bullet \mathrm{C}^{21} & & & \\ \mathrm{G}^{5} \bullet \mathrm{C}^{21} & 11.68 & 6.82 & 8.10 \\ \mathrm{C}^{6} \bullet \mathrm{G}^{19} & 13.01 & 6.56 & 8.38 \\ \mathrm{G}^{7} \cdot \mathrm{C}^{18} & 13.17 & 6.60 & 8.38 \\ \mathrm{C}^{8} \bullet \mathrm{G}^{17} & 13.13 & 6.56 & 8.36 \\ \mathrm{C}^{9} \bullet \mathrm{G}^{16} & 12.99 & 7.05 & 8.71 \\ \mathrm{~A}^{10} \cdot \mathrm{T}^{15} & 13.82 & & \\ \mathrm{~T}^{11} \bullet \mathrm{A}^{14} & 13.97 & & \end{array}$

NarIIQ2 base pair G N1H/T N3H C/X N4Ha amino C/X N4Hb amino

$\begin{array}{llll}\mathrm{T}^{2} \bullet \mathrm{A}^{23} & 14.21 & & \\ \mathrm{C}^{3} \cdot \mathrm{G}^{22} & 13.03 & 6.58 & 8.33 \\ \mathrm{G}^{4} \bullet \mathrm{C}^{21} & 12.52 & 6.22 & 7.65 \\ \mathrm{X}^{5} \cdot \mathrm{C}^{21} & & & \\ \mathrm{C}^{6} \cdot \mathrm{G}^{19} & 12.18 & 6.50 & 8.32 \\ \mathrm{G}^{7} \cdot \mathrm{C}^{18} & 12.96 & 6.56 & 8.30 \\ \mathrm{C}^{8} \cdot \mathrm{G}^{17} & 13.05 & 6.56 & 8.36 \\ \mathrm{C}^{9} \cdot \mathrm{G}^{16} & 13.00 & 7.06 & 8.64 \\ \mathrm{~A}^{10} \bullet \mathrm{T}^{15} & 13.85 & & \\ \mathrm{~T}^{11} \bullet \mathrm{A}^{14} & 14.03 & & \end{array}$


Table S3. Comparison of Experimental Intermolecular Distance Restraints with those Observed for Intensity-Refined Structures of the NarIIQ1 and NarIIQ2 Duplexes.

Interproton distances $(\AA)$

Exptl bounds

Calculated

\begin{tabular}{|c|c|c|}
\hline \multicolumn{3}{|l|}{ NarIIQ1 } \\
\hline IQ H9A-C ${ }^{20} \mathrm{H} 2^{\prime}$ & $2.58-3.98$ & 3.25 \\
\hline IQ H9A-C ${ }^{20} \mathrm{H} 2^{\prime \prime}$ & $3.58-4.98$ & 4.07 \\
\hline IQ H9A-G ${ }^{22}{ }^{4} 4^{\prime}$ & $4.83-6.33$ & 5.10 \\
\hline IQ H9A-C ${ }^{21} \mathrm{H} 4^{\prime}$ & $4.10-6.00$ & 5.81 \\
\hline IQ H9A-C ${ }^{20} \mathrm{H}^{\prime}$ & $4.80-6.30$ & 5.20 \\
\hline IQ H9A-C ${ }^{21} \mathrm{H} 1^{\prime}$ & $3.20-4.70$ & 4.42 \\
\hline IQ H9A-C ${ }^{20} \mathrm{H} 1^{\prime}$ & $4.20-5.70$ & 4.93 \\
\hline IQ H9A-G ${ }^{22}$ H8 & $4.63-6.13$ & 6.15 \\
\hline IQ H9A-C ${ }^{20}$ H6 & $4.23-5.73$ & 5.75 \\
\hline IQ H4A-G ${ }^{22} \mathrm{H} 1^{\prime}$ & 4.09-6.09 & 4.90 \\
\hline IQ H5A-G ${ }^{22} \mathrm{H} 1^{\prime}$ & $3.80-5.30$ & 5.30 \\
\hline IQ CH3-G $\mathrm{G}^{5} 1^{\prime}$ & $3.64-5.65$ & 4.67 \\
\hline IQ CH3-X4 $\mathrm{H}^{\prime}$ & $3.58-5.56$ & 3.59 \\
\hline IQ CH3-G ${ }^{5} 5^{\prime}$ & $2.74-4.24$ & 3.51 \\
\hline IQ CH3-G $\mathrm{G}^{5} \mathrm{H}$ & $4.00-6.00$ & 5.72 \\
\hline IQ CH3-G ${ }^{22} \mathrm{H} 1$ & $2.50-4.50$ & 3.52 \\
\hline \multicolumn{3}{|l|}{ NarIIQ2 } \\
\hline IQ H9A-G ${ }^{19} \mathrm{H} 1^{\prime}$ & $2.98-4.04$ & 3.81 \\
\hline IQ H9A-G ${ }^{19} \mathrm{H} 2^{\prime \prime}$ & $2.98-5.79$ & 3.15 \\
\hline IQ H9A-G ${ }^{19} \mathrm{H} 3^{\prime}$ & $3.33-5.48$ & 5.44 \\
\hline
\end{tabular}




\begin{tabular}{|c|c|c|}
\hline IQ H9A-G ${ }^{19} \mathrm{H} 8$ & 2.54-4.19 & 4.01 \\
\hline IQ H9A-C ${ }^{21}$ H6 & $4.05-6.57$ & 6.50 \\
\hline IQ H9A-C ${ }^{20} \mathrm{H} 1^{\prime}$ & $4.84-6.34$ & 5.05 \\
\hline IQ H8A-G ${ }^{19} \mathrm{H} 8$ & $2.97-4.24$ & 4.02 \\
\hline IQ H8A-C ${ }^{20} \mathrm{H} 2^{\prime \prime}$ & $3.81-5.79$ & 5.72 \\
\hline IQ H5A-C ${ }^{21} \mathrm{H}^{\prime}$ & $2.83-4.33$ & 3.76 \\
\hline IQ H5A-C ${ }^{20} \mathrm{H} 2^{\prime \prime}$ & $2.61-4.94$ & 4.24 \\
\hline IQ $\mathrm{H} 5 \mathrm{~A}-\mathrm{C}^{20} \mathrm{H} 2^{\prime}$ & $2.83-5.23$ & 5.20 \\
\hline IQ H5A-C ${ }^{21} H 4^{\prime}$ & $3.01-5.98$ & 3.99 \\
\hline IQ H5A-C ${ }^{21} \mathrm{H} 5$ & $3.25-6.09$ & 6.00 \\
\hline IQ H4A-C ${ }^{21} \mathrm{H} 1^{\prime}$ & $2.25-3.30$ & 3.29 \\
\hline IQ $\mathrm{CH}_{3}-\mathrm{C}^{6} \mathrm{H} 1^{\prime}$ & $2.24-3.44$ & 2.68 \\
\hline IQ $\mathrm{CH}_{3}-\mathrm{X}^{5} \mathrm{H} 1^{\prime}$ & $3.84-6.21$ & 4.13 \\
\hline IQ $\mathrm{CH}_{3}-\mathrm{C}^{21} \mathrm{H} 1^{\prime}$ & $4.16-5.62$ & 4.26 \\
\hline IQ $\mathrm{CH}_{3}-\mathrm{C}^{6} \mathrm{H} 4^{\prime}$ & $2.11-3.81$ & 3.32 \\
\hline IQ $\mathrm{CH}_{3}-\mathrm{G}^{4} \mathrm{H} 1$ & $2.53-4.53$ & 3.66 \\
\hline IQ $\mathrm{CH}_{3}-\mathrm{G}^{19} \mathrm{H} 1$ & $4.03-6.03$ & 5.70 \\
\hline
\end{tabular}


Wang, F., et al.

Table S4. Pseudorotation Angles and Glycosyl Torsion Angles for the Structures of the Central $5^{\prime}-\mathrm{d}\left(\mathrm{G}^{4} \mathrm{G}^{5} \mathrm{C}^{6} \mathrm{X}^{7} \mathrm{C}^{8} \mathrm{C}^{9}\right)-3^{\prime} \cdot 5^{\prime}-\mathrm{d}\left(\mathrm{G}^{16} \mathrm{G}^{17} \mathrm{C}^{18} \mathrm{G}^{19} \mathrm{C}^{20} \mathrm{C}^{21}\right)-3^{\prime}$ Segments of the Refined Structures of the NarIIQ1 and Modified NarIIQ2 duplexes.

NarIIQ1

Nucleotide $\chi$

$\mathrm{X}^{4} / \mathrm{G}^{4}$

$\mathrm{G}^{5} / \mathrm{X}^{5}$

$\mathrm{C}^{6}$

$\mathrm{G}^{7}$

$\mathrm{C}^{8}$

$\mathrm{C}^{9}$

$\mathrm{C}^{21}$

$\mathrm{C}^{20}$

$\mathrm{G}^{19}$

$\mathrm{C}^{18}$

$\mathrm{G}^{17}$

$\mathrm{G}^{16}$
123

246

234

233

232

238

264

216

236

239

248

245
P

149

148

123

150

123

128

124

114

150

110

150

157
NarIIQ2

$\chi$

231

110

229

251

238

237

228

101

218

225

248

242

246
117

119

143

126

150

163

139

122

148

124

149 
Figure S1. Expanded DQF-COSY contour plot in $\mathrm{D}_{2} \mathrm{O}$ buffer at $25^{\circ} \mathrm{C}$ establishing coupling between the $\mathrm{H}^{\prime}$ and $\mathrm{H}^{\prime}, \mathrm{H}^{\prime \prime}$ protons. The $\mathrm{H} 2^{\prime}$ and $\mathrm{H} 2^{\prime \prime}$ protons of the residues around the lesion site are connected by lines and labeled. (A). The NarIIQ1 duplex. (B). The NarIIQ2 duplex.

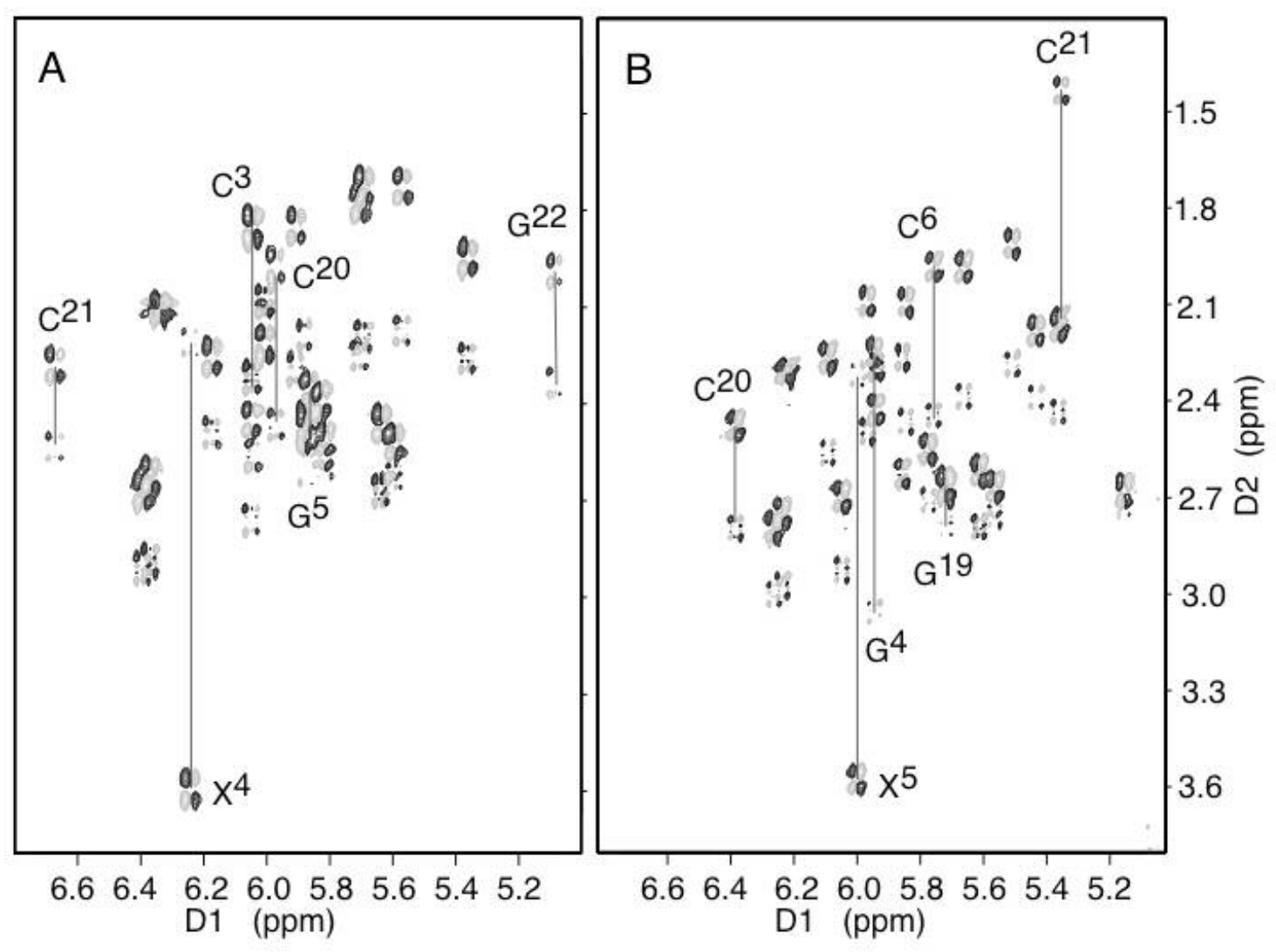


Figure S2. The H3' region of non-selective excitation ${ }^{31} \mathrm{P}-{ }^{1} \mathrm{H}$ HMBC spectra. A.

Unmodified duplex. B. The NarIIQ1 duplex. C. The NarIIQ2 duplex. All spectra were collected at $298 \mathrm{~K}$.
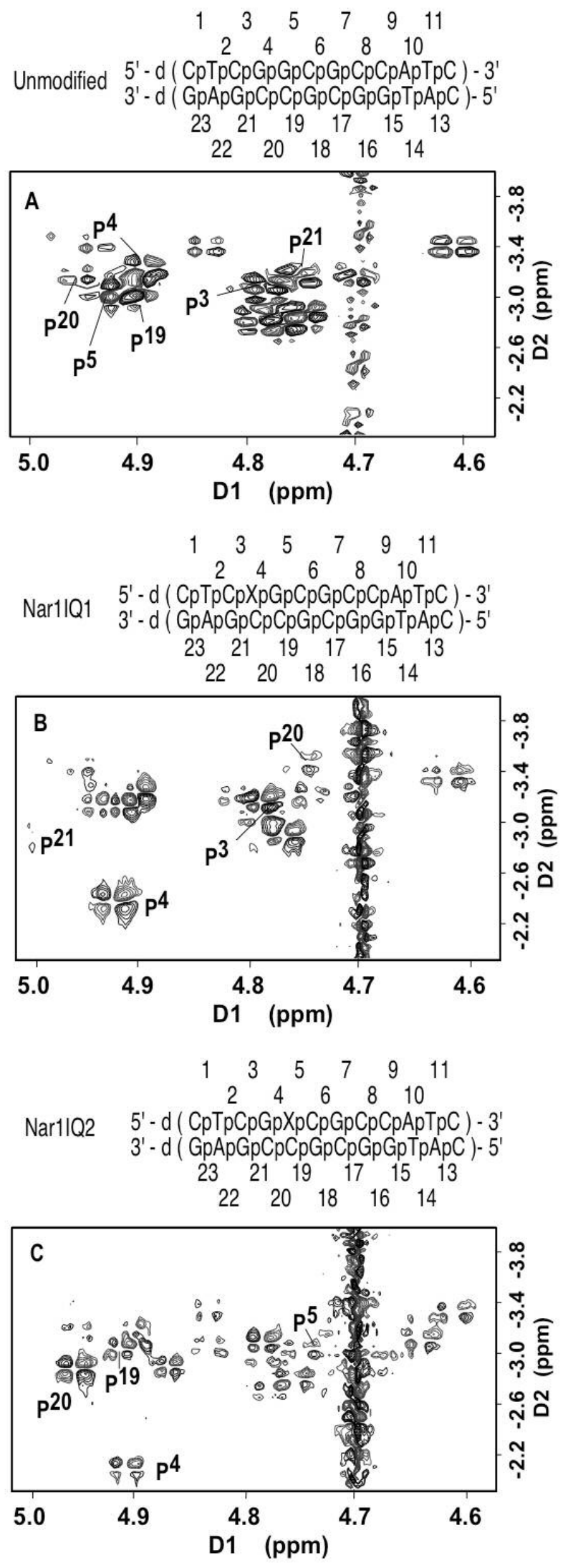
Figure S3. (A) Expanded plots from the COSY spectrum and (B) the NOESY spectrum at $15{ }^{\circ} \mathrm{C}$ for IQ-adducted RasIQ5, showing assignments for the IQ protons.

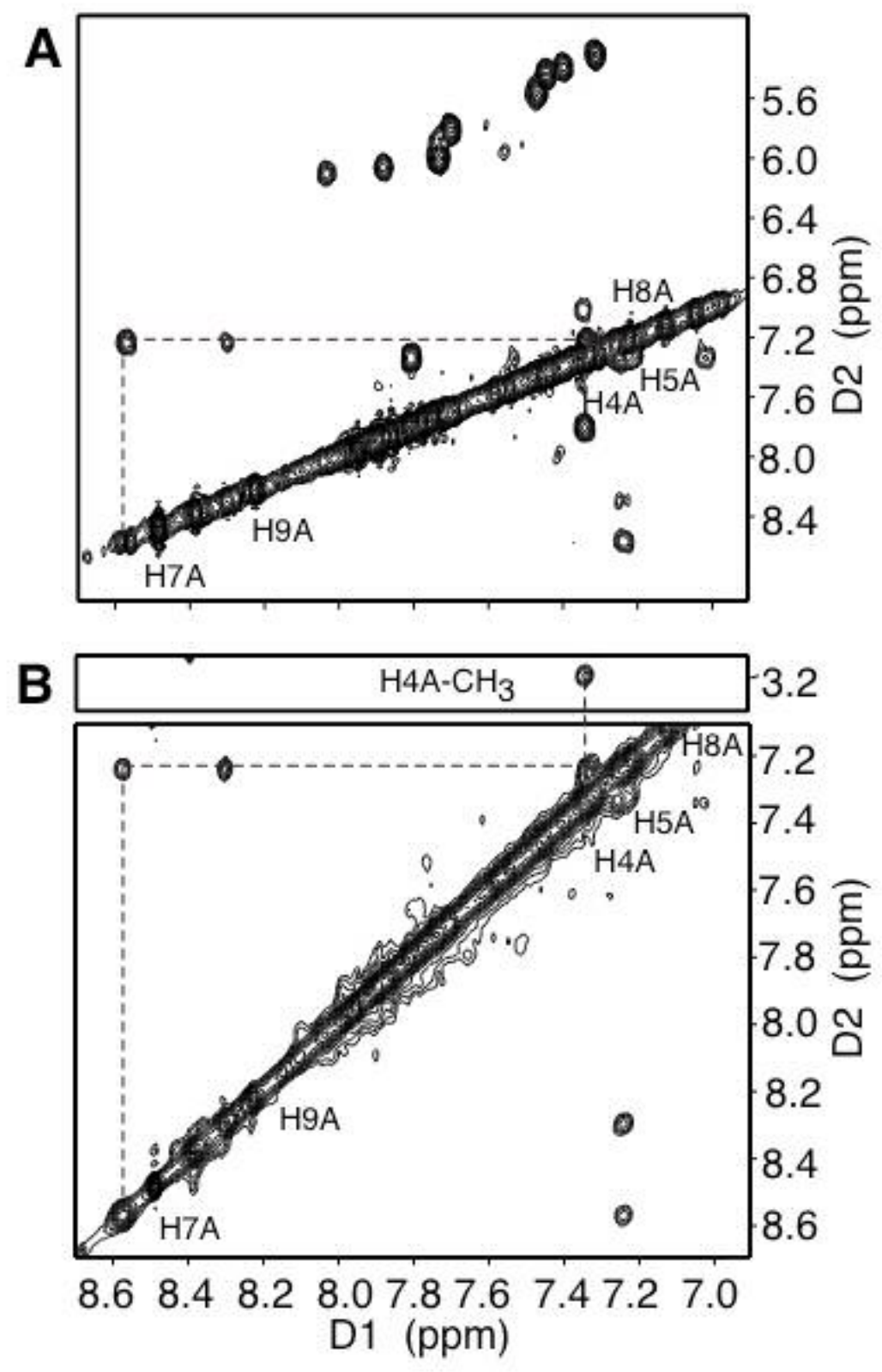


Figure S4. Expanded COSY contour plot for the RasIQ5 duplex in $\mathrm{D}_{2} \mathrm{O}$ buffer at $15{ }^{\circ} \mathrm{C}$ establishing coupling connectivity between the $\mathrm{H}^{\prime}$ and $\mathrm{H}^{\prime}, \mathrm{H}^{\prime \prime}$ protons.

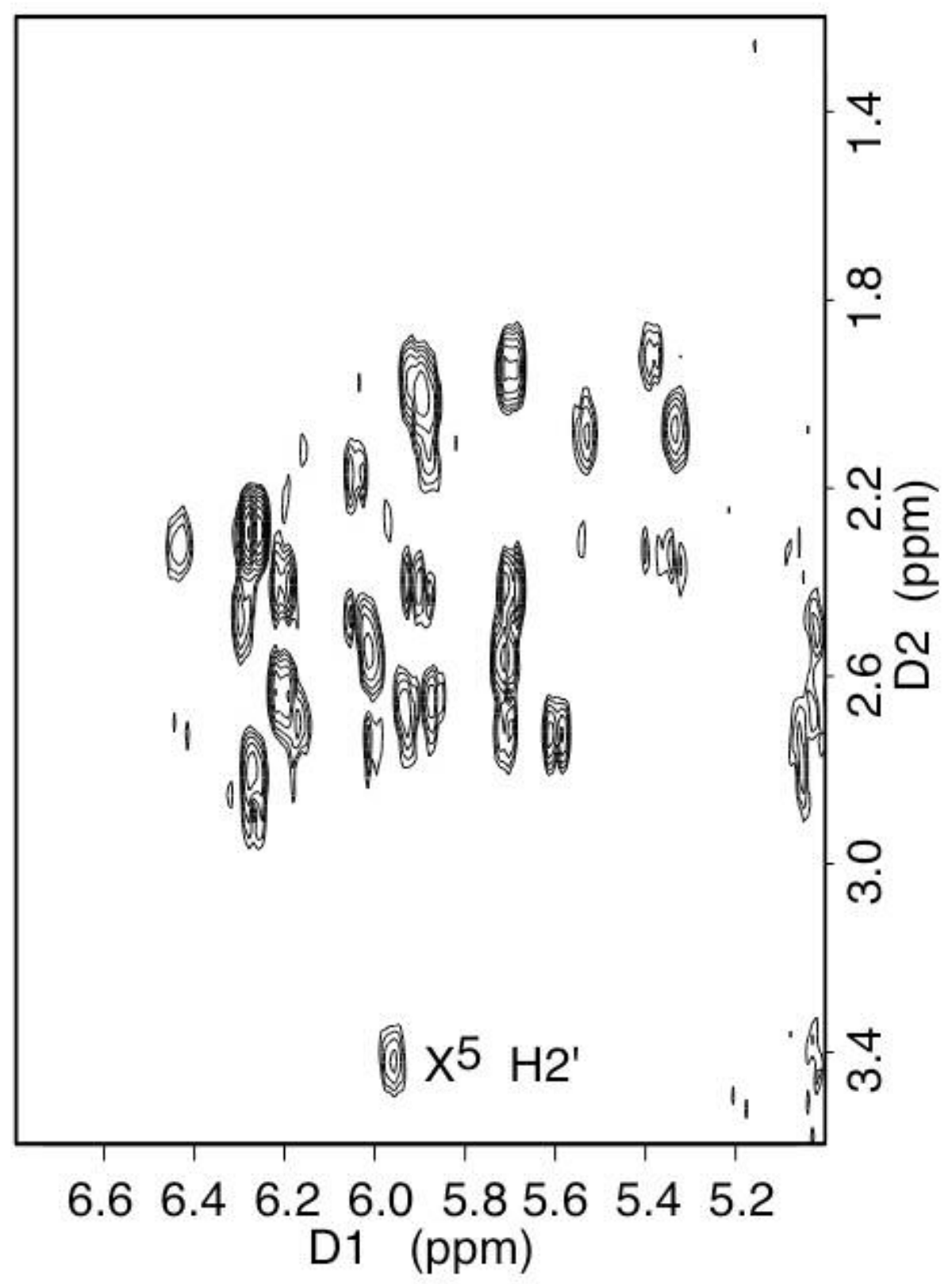

